\title{
Prospettive ovidiane per la città imperiale
}

Nel poema sul calendario romano, Ovidio sembra perseguire una strategia di retrocessione cronologica: alla ricerca di eziologie c'è sempre un passo indietro da compiere verso il passato più remoto. Il punto di arrivo è l'occupazione del tempo delle origini umane: solo con la conquista poetica del tempo, al di là dei confini della storia, la civiltà romana può acquisire una compiuta identità come civiltà universale, adeguata alla capitale di un impero ecumenico, prendendo al tempo stesso coscienza di quella pluralità di valori, di ideologie, e di immagini della vita, che una identità fondata sulla storia aveva finito per costringere nelle angustie del mos maiorum. ${ }^{\mathrm{I}}$

Il personaggio di Evandro gioca un ruolo di primo piano nella strategia universalistica dei Fasti: ${ }^{2}$ l'eroe arcade non soltanto giunge in Italia come Enea, ma come Enea porta con sé sulla sua nave delle divinità che, nel momento in cui egli si insedia nel sito di Roma identificata come locus imperii, diventano elementi caratterizzanti l'identità imperiale di Roma stessa.

E' interessante riflettere sulle caratteristiche dei sacra che Ovidio mette in rilievo tra quelli che una consolidata tradizione annalistica e antiquaria attribuiva all'apporto di Evandro, in particolare il culto di Fauno, che trova la sua espressione più impressionante nei Lupercalia:

Ov. fast. 5,99-104

sacraque multa quidem, sed Fauni prima bicornis

has docuit gentes alipedisque dei.

semicaper, coleris cinctutis, Faune, Lupercis,

cum lustrant celebres verbera secta vias;

at tu materno donasti nomine mensem,

inventor curvae, furibus apte, fidis.

I Lupercalia erano uno dei sacra più spettacolari, pittoreschi e violenti dell'anno romano, che Augusto aveva restaurato, cercando al tempo stesso di metterne sotto controllo gli eccessi: 3 la corsa sfrenata dei Luperci nudi, la flagellazione soprattutto delle donne con il cuoio ancora sanguinante del capro immolato, probabilmente per favorirne magicamente la fecondità. Un rituale che mette in contatto la modernità augustea col mondo primitivo delle origini della città, con quel primo padre della patria che aveva dovuto assicurare, in

I Riprendo qui considerazioni da me svolte e argomentate in Labate (20I0), soprattutto I92Ss., 2IOss.

2 Fantham (I992) I55ss.; Barchiesi (I994) I85 ss.; Labate (2010) I64Ss.

3 Fraschetti (I990) 25ISs. 
maniera adeguata alla rudezza dei tempi, la propagazione della sua gente: prima procurando donne ai suoi soldati senza troppo badare alle regole del vivere civile (il ratto delle Sabine), ${ }^{4}$ poi scongiurandone la sterilità con l'esecuzione 'attenuata' dello sconcertante oracolo di Giunone Lucina che le voleva 'penetrate' dal sacro caprone (fast. 2,44I Italidas matres ... sacer hircus inito). ${ }^{5}$

Nell'immaginario della generazione che aveva vissuto il periodo tumultuoso dell'ultima repubblica il carattere selvaggio e primitivo del rituale connesso alle confraternite dei Luperci si riattualizzava nei disordini e nelle violenze politiche che insanguinavano le strade di Roma, rendendo sempre più inquieto il presente e fosco il futuro. Cicerone commentava ironicamente il fatto che Erennio, l'accusatore di Celio, avesse tirato in ballo, nell'accusa, qualche fatto connesso alla comune appartenenza alla confraternita dei Luperci: ${ }^{6}$

Cic. Cael. 26

Neque vero illud me commovet quod sibi in Lupercis sodalem esse Caelium dixit. Fera quaedam sodalitas et plane pastoricia atque agrestis germanorum Lupercorum, quorum coitio illa silvestris ante est instituta quam humanitas atque leges, si quidem non modo nomina deferunt inter se sodales sed etiam commemorant sodalitatem in accusando, ut ne quis id forte nesciat timere videantur!

Ma era soprattutto l'episodio delle idi di febbraio del 44 a collegare la corsa dei Luperci al disordine tumultuoso che metteva a repentaglio lo stato, rendendo verosimile che la comunità romana rinunciasse ai suoi ordinamenti, alla legalità repubblicana gelosamente custodita post reges exactos, per riconsegnarsi nelle mani di un potere tirannico e arbitrario. Durante la festa, il console luperco Antonio, a capo della nuova confraternita dei Luperci Iulii, che il dittatore aveva aggiunto nel suo nome alle due sodalitates dei Fabii e dei Quinctiales, si era staccato dall'abituale percorso della corsa sfrenata dei giovani nudi muniti di corregge sanguinanti, per salire fino ai rostri e offrire a Cesare il diadema del potere regale.7 Quel gesto e quel diadema divennero facilmente simbolo di di un mondo sregolato e primitivo in cui tutto si poteva osare "prima che fossero istituite la civiltà e le leggi". Consumatesi nel sangue delle idi di marzo quelle prospettive e quelle paure, sono significative le cautele con cui Augusto aveva ripristinato, fra i tanti relitti della religione patria, quell'antichissimo rito: era stata restaurata la grotta del Lupercal, la corsa dei Luperci era stata reinserita nell'ambito della festa delle idi di febbraio, ma la confraternita legata al nome di Cesare e alle tentazioni violente della gioventù romana venne lasciata cadere. Anzi il principe si preoccupò di ricondurre la confraternita in un contesto sociale ordinato e controllato, riservandone l'accesso ai membri di quell'ordine equestre, che, come ha osservato A. Fraschetti, alle idi di luglio

4 Labate (2006).

5 Labate (2010) I84.

6 Cf. Holleman (1975).

7 La bibliografia è molto ampia: per restare solo ai contributi più recenti, vedi Fraschetti (I990) 252; Zecchini (200I) IISs.; North (2008); Cristofoli (2008) I40ss. (tutti con ulteriori indicazioni). 
sfilava ordinatamente, attraverso il Circo massimo e il Foro, nella solenne e grandiosa parata della transvectio equitum che Dionigi di Alicarnasso giudicava degna della grandezza

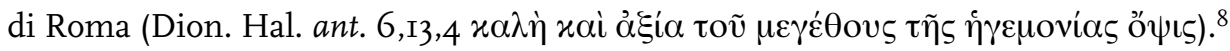

Nella complessa eziologia greco-latina della corsa dei Luperci e della nudità dei Luperci, 9 Ovidio fa riferimento al demone arcadico della natura selvaggia che, percorrendo di corsa le vette montane, concepisce e ispira fughe improvvise e irrazionali ( $\pi \alpha v$ ixòs

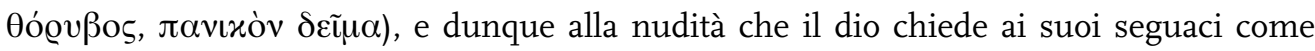
opportuna alla corsa, oppure alla primitività degli Arcadi come testimoni del tempo antichissimo in cui gli uomini delle origini vivevano a cielo aperto, senza alcuna veste che riparasse il loro corpo dalla pioggia e dal vento, oppure ancora all'esotismo della "sexual comedy" che ha come protagonisti Ercole, Onfale e Fauno, in cui il dio dal nome già italico agisce nell'Asia Minore della mollezza e della trasgressività. ${ }^{\text {IO }}$

La causa latina che viene messa in concorrenza, o meglio in parallelo, con le causae peregrinae, la storia nazionale di Romolo, Remo e i predoni, condisce un generale colore primitivistico-pastorale con qualche divertito ammiccamento anacronistico. ${ }^{\text {II }}$ Il quadro ovidiano si distingue però per qualche particolare non irrilevante: manca ogni sottolineatura della durezza primitiva; in compenso, l'episodio sembra ambientato nel soleggiato campo di Marte, suggerendo uno scenario che agli occhi dei contemporanei evocava immagini di vita urbana ben poco primitiva: Ovidio ha insomma trovato il modo di coniugarlo con l'aition delle ricreazioni urbane della gioventù augustea. ${ }^{\mathrm{I2}}$

Mentre Romolo, Remo e gli altri giovani sono impegnati in questi esercizi, un pastore da una posizione di vedetta dà l'allarme, avvertendo che i praedones stanno portandosi via il bestiame in luoghi remoti e inaccessibili. Non c'è tempo di armarsi e i giovani divisi in due squadre, l'una al comando di Romolo, l'altra al comando di Remo, si lanciano in direzione opposta alla ricerca dei briganti, così come si trovano, nudi per gli esercizi ginnici. Il successo arride alla schiera di Remo che s'imbatte nei predoni, recupera la preda e ritorna alla base prima di Romolo e dei suoi Quintilii, consumando tutte le carni del sacrificio, nel frattempo cotte a puntino, come appannaggio dei vincitori, senza lasciarne alla squadra del fratello. Quando arriva anche Romolo e non trova che ossa spolpate, la sua reazione esprime il rammarico per il successo del fratello ma al tempo stesso prospetta, con una risata, il possibile assorbimento non conflittuale della contesa:133

8 Fraschetti (I990) 253, con indicazioni bibliografiche.

9 Cf. Holleman (I973); Littlewood (I975); Newlands (I995) 59ss.

IO Fantham (1983); Barchiesi (1994) 226ss.

II Barchiesi (I994) I44Ss.

I2 Labate (20I0) I82 n.I.

I3 Una diversa, 'amara' interpretazione del riso di Romolo come sintomo di doppiezza e annuncio di una prospettiva più inquietante e minacciosa in Barchiesi (1994) I44ss. 


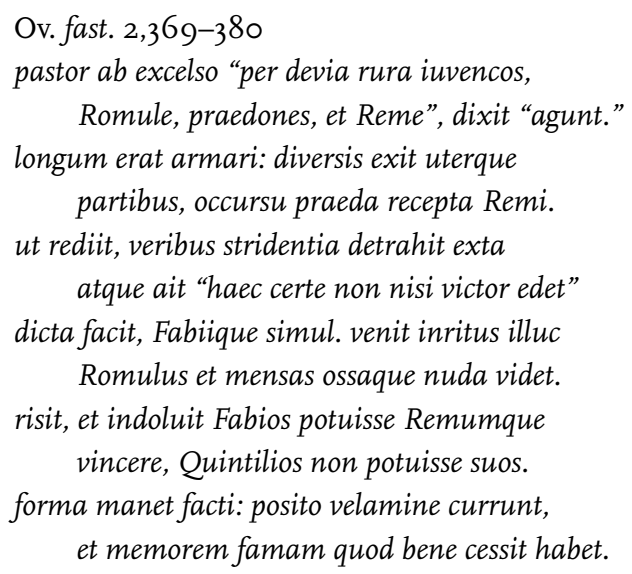

Il racconto di Livio presenta una versione sensibilmente diversa. La cultura antica associava la vita del pastore a un'esistenza in spazi marginali e selvaggi, in cui gli uomini dediti all'allevamento del bestiame potevano essere vittima o protagonisti di violenze e di attività brigantesche. ${ }^{\mathrm{I} 4}$ I gemelli raccolti da Faustolo rivelano un'indole incline a un modello energico e combattivo di pastore-cacciatore e pastore-predone, rivale di predoni, più che a un modello di pastore intento a governare pacificamente il bestiame e le stalle:

Liv. I,4,8-9

Ita geniti itaque educati, cum primum adoleuit aetas, nec in stabulis nec ad pecora segnes uenando peragrare saltus. hinc robore corporibus animisque sumpto iam non feras tantum subsistere sed in latrones praeda onustos impetus facere pastoribusque rapta diuidere et cum his crescente in dies grege iuuenum seria ac iocos celebrare.

Nell'ambito del culto arcadico di Pan Lycaeus, introdotto sul Palatino da Evandro, si celebra il rituale della corsa sfrenata di giovani nudi, cui partecipano Romolo Remo e il crescente grex pastorum che i gemelli vanno aggregando intorno a sé attraverso le distribuzioni delle prede strappate ai predoni. I latrones cui i gemelli avevano sottratto la preda approfittano della situazione tendendo un agguato nel corso del quale Romolo riesce a difendersi mentre Remo viene preso, consegnato al re Amulio e accusato preventivamente di brigantaggio. Romolo e Remo sono presentati come dei violenti che danno l'assalto alle terre di Numitore per reclutare bande di giovani da guidare in scorrerie brigantesche: la consegna del presunto brigante Remo nelle mani di Numitore stesso per la punizione è poi l'occasione per il riconoscimento dei gemelli, l'uccisione dell'usurpatore Amulio, il reinsediamento di Numitore sul trono di Alba e la successiva iniziativa della nuova città da fondare nel luogo in cui erano stati esposti e allevati. 
E' evidente che, nella versione di Livio, la storia non è direttamente collegata all'origine dei Lupercalia (la corsa dei giovani nudi infatti esiste già, in connessione alla sua causa peregrina, l'origine arcadica), quanto piuttosto all'origine stessa della città. Livio inserisce l'episodio in quel mondo violento delle origini in cui Romolo e Remo sono troppo poco diversi dai latrones con cui competono e portano in sé quel germe del male ereditario della contesa fraterna per il potere (I, 6, 4 avitum malum, regni cupido) che aveva opposto Amulio a Numitore e che porterà alla contesa tra i fondatori di Roma, al fratricidio, al seme delle guerre civili.

Ovidio ha invece rappresentato, nell'episodio di Romolo, Remo e i predoni, l'aition di una cerimonia che serba memoria di una componente primitiva della città imperiale, ma di una primitività che non solo sa convivere con una modernità anacronisticamente richiamata (le esercitazioni sportive della gioventù augustea al Campo di Marte), ma esprime già una capacità di contrasto e di controllo di quella violenza e di quell'arbitrio che appartengono alle origini, ma sono sempre in agguato nella storia, come la storia recente, con le sue bande di latrones, aveva a tutti ricordato. La corsa dei Luperci, nell'aition romano valorizzato da Ovidio, finisce insomma per proporre un modello di ben riuscita azione di forze dell'ordine impegnate nel controllo del territorio:

Ov. fast. $2,379-380$

Forma manet facti: posito velamine currunt, et memorem famam quod bene cessit habet.

Se, con i sacra connessi a Pan-Fauno, Evandro ha introdotto a Roma un mondo religioso che affonda le radici nel mondo primitivo dei pastori, delle scorrerie violente, del furto di bestiame, quelli che si riferiscono all'altra divinità arcade, Mercurio, ci mostrano come la città imperiale sia anche il luogo della più spregiudicata modernità, capace di imporre modelli e valori in netto contrasto con la moralità della tradizione ancestrale agraria.

Nato dall'unione di Giove con la più bella delle Pleiadi sulle pendici boscose del Cillene, il dio si presenta nella dimensione della leggerezza, dell'agilità e del dinamismo, come quello che con i suoi piedi alati percorre le vie del cielo ed è capace di dominare in lungo e in largo spazi e territori sconfinati. Il mese di maggio, al di là delle molteplici possibili attribuzioni del nome, accoglie, nel giorno delle Idi, la festa del dio arcadico, la cui trattazione da parte del poeta del calendario è chiaramente strutturata in tre blocchi: una specie di inno a Mercurio ex persona poetae, la descrizione di un cerimonia (notturna?) di purificazione delle merci e del mercante con l'acqua attinta all'aqua Mercurii che sgorgava nei pressi di porta Capena, la preghiera a Mercurio che Ovidio fa pronunciare al mercante stesso nell'ambito del rito di purificazione.

Le attribuzioni del dio sono illustrate in una specie di inno cletico che si pone in evidente rapporto intertestuale con l'ode I, so di Orazio, l'inno a Mercurio del poeta lirico di Roma. 
Ov. fast. 5, 663-672

Clare nepos Atlantis, ades, quem montibus olim edidit Arcadiis Pleias una Iovi:

pacis et armorum superis imisque deorum arbiter, alato qui pede carpis iter,

laete lyrae pulsu, nitida quoque laete palaestra, quo didicit culte lingua docente loqui,

templa tibi posuere patres spectantia Circum Idibus; ex illo est haec tibi festa dies.

te, quicumque suas profitentur vendere merces, ture dato tribuas ut sibi lucra rogant.

Hor. carm. I,IO

Mercuri, facunde nepos Atlantis,

qui feros cultus hominum recentum

voce formasti catus et decorae more palaestrae,

te canam, magni Iovis et deorum

nuntium curvaeque lyrae parentem, callidum quidquid placuit iocoso condere furto.

te, boves olim nisi reddidisses per dolum amotas, puerum minaci voce dum terret, viduus pharetra risit Apollo.

quin et Atridas duce te superbos Ilio dives Priamus relicto Thessalosque ignis et iniqua Troiae castra fefellit.

tu pias laetis animas reponis sedibus virgaque levem coerces aurea turbam, superis deorum gratus et imis.

La confrontabilità di questi due testi, garantita dalla memoria incipitaria (facunde nepos Atlantis / clare nepos Atlantis), si dipana attraverso una serie di elementi comuni:15 Mercurio dio della parola civilizzatrice e dell'eloquenza (facunde .../qui feros cultus hominum recentum) voce formasti catus, in Orazio; quo didicit culte lingua docente loqui, in Ovidio); Mercurio

I5 Cf. Miller (I993) I6oss. (= Miller [2006] 95ss.). 
inventore della lira e della palestra: musica e ginnastica, due componenti fondamentali della civilizzazione secondo la paideia greco-romana (decorae more palaestrae ... curvae lyrae parentem, in Orazio; laete lyrae pulsu, nitida quoque laete palaestrae, in Ovidio); Mercurio come nuntius deorum e come gestore del contatto e della comunicazione tra sfere distinte o contrapposte: tra amici e nemici, mondo supero e mondo infero (Orazio fa riferimento al mito omerico dell'accompagnamento di Priamo al campo greco per trattare la restituzione del corpo di Ettore e alla funzione dello psicopompo che accompagna le anime nell'oltretomba; Ovidio preferisce dilatare questo ruolo del dio in una dimensione cosmica "arbitro della pace e della guerra per gli dei del cielo e per quelli degli inferi”, ma superis imisque deorum è una citazione del finale dell'ode oraziana superis deorum gratus et imis.

Questa evidente intertestualità impone di valorizzare le differenze, che risiedono soprattutto nel modo diverso in cui i due poeti fanno riferimento a un tema inevitabile nella caratterizzazione del ruolo del dio nel pantheon greco-latino: Hermes-Mercurio come dio che rappresenta e garantisce la sfera della metis, dell'intelligenza astuta, della versatilità, della duttilità di un pensiero che sa adattarsi alle circostanze, dell'agilità e della rapidità con cui l'occasione fortunata viene colta nel momento in cui si presenta o viene costruita con sofisticate strategie. ${ }^{16}$ L'abilità, la destrezza, la mente acuta di Hermes si associano a una certa spregiudicatezza. L'astuzia, la sfrontatezza e perfino la mancanza di scrupoli è il modo in cui può essere ottenuto un successo difficile, in qualche modo inaspettato, perché si realizza spesso in condizioni di svantaggio, di evidente disparità di forze e di risorse. La figura di Hermes-Mercurio ${ }^{17}$ rappresenta, nella cultura religiosa e nella mitologia greco-romana, un modello culturale e antropologico alternativo a quello della arete/virtus, quello stesso modello che trovava espressione nel personaggio di Ulisse, l'eroe polytropos/versutus per eccellenza, collegato al dio da un legame che è al tempo stesso genealogico (HermesAutolico-Anticlea-Ulisse) e tipologico. ${ }^{18}$

Nell'inno omerico a Hermes è centrale il motivo del dio-neonato (quale condizione di apparente debolezza maggiore di questa?) che, con audacia e sofisticati stratagemmi, ruba le giovenche di Apollo, affermandosi come prototipo dei furfanti e dei ladri, ${ }^{19}$ ed è poi capace di tener testa all'ira del dio tanto più potente e minaccioso con la sfrontatezza del mentitore e dello spergiuro. Maia genera a Zeus "un figlio dalle molte arti, dalla mente sottile, predone, ladro di buoi, ispiratore di sogni, vigile nella notte, che sta in agguato alle porte ... Nato all'aurora, a mezzogiorno suonava la lira e dopo il tramonto rubò le vacche di Apollo arciere" (vv. I3-I8). Il suo piano è "un inganno fuori del comune, quali ne preparano i ladri nel corso dell'oscura notte" $2 \circ$ (v. 67); è l'“arte dell'inganno" che gli ispira il modo ingegnoso di confondere le tracce. Come poikilometes, come uno che arriva "nel cuore della

\footnotetext{
I6 Ancora fondamentale Detienne-Vernant (I974).

I7 Cassola (I975), I53ss.; Combet Farnouz (I980) e (I98I).

I8 Stanford (I954); Perutelli (2006).

I9 Cassola (I975) I6oss.

20 Trad. di F. Cassola.
} 
notte, rivestito di impudenza", il fanciullo viene apostrofato dalla madre Maia. E il fanciullino le risponde esponendo sfrontatamente il suo progetto: ottenere per se e per la madre uno status paragonabile a quello degli altri dei olimpici e di Apollo in particolare: "se poi mio padre non me li darà, in verità io, per mio conto, mi adopererò per diventare il re dei ladri: ne sono capace" (I74-I75).

Di fronte alle menzogne spudorate e agli spergiuri del fanciullino, Apollo gli predice ridendo un futuro degno di questi inizi: "O caro ingannatore e imbroglione, credo in verità che spesso tu, penetrando nelle dimore ben costruite, in piena notte, più d'un uomo lascerai sulla nuda terra, saccheggiando per tutta la casa senza rumore ... questo privilegio, senza dubbio, avrai anche in futuro fra gl'immortali: sarai chiamato per sempre re dei furfanti

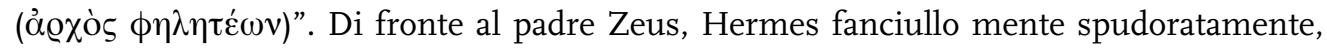
tanto più che esordisce con improbabili dichiarazioni di sincerità: "dirò la verità: sincero infatti io sono e non so mentire", il tutto suggellato dal più solenne degli spergiuri. Zeus ride divertito e quasi ammirato dell'abilità e della sfrontatezza del suo intrigante figliolo.

L'inno omerico non sembra conoscere invece il motivo dell'ulteriore tiro mancino, il furto della faretra, che il fanciullo gioca ad Apollo proprio nel momento in cui questi lo sta minacciando di punizioni terribili, nel caso che il ladro non restituisca le vacche dolosamente sottratte, anche se il rapsodo fa esprimere al dio, nell'ambito della riappacificazione con Hermes e dello scambio di concessioni reciproche, il timore che quel poco rassicurante partner "possa rubargli la cetra o l'arco ricurvo" (516).

Proprio su questo motivo Orazio costruisce la strofe centrale del suo inno, in cui la prerogativa di Mercurio come dio dei ladri si declina secondo un paradigma probabilmente desunto dall'inno di Alceo di cui conserviamo l'esordio, ma da cui abbiamo notizia (da Porfirione $)^{2 \mathrm{I}}$ che Orazio mutuasse la scena del furto dell'arco o della faretra, notizia che trova qualche conferma in uno scolio all' Iliade $^{22} \mathrm{e}$ in un passo di Filostrato: ${ }^{23}$ il furto si consumava in Alceo e si consuma in Orazio come manifestazione di una intelligenza astuta e giocosa (callidum, quicquid placuit, iocoso/condere furto), di una irrefrenabile vitalità che suscita sorpresa, ammirazione e divertimento, in uno spirito che deriva dalla leggerezza della cultura ionica, ma che è reinterpretabile nella grazia del gusto letterario e figurativo alessandrino (si pensi in particolare al ruolo delle impertinenti divinità fanciulle, a cominciare da Eros, nella poesia e nell'arte ellenistica).

Questa interpretazione del furto come libero gioco dell'intelligenza creativa, arte fine a se stessa, liberata dalla imbarazzante ipoteca del furto come appropriazione di un bene, dettato dal desiderio di guadagno e di ricchezza materiale, era più agevolmente conciliabile con l'intepretazione filosofico-allegorica della figura di Mercurio come creatore di civiltà e dio del logos e del linguaggio, che Orazio sottolineva nella prima parte dell'ode e

$2 \mathrm{I}$ Porph. Hor. c. I,IO,II.

22 Schol. D in Hom. Iliad. I5,256.

23 Philostr. imag. I,26. Tutti I passi utili sono convenientemente raccolti nel commento di Nisbet-Hubbard (I970). 
che imponeva soprattutto una vistosa omissione, omissione che risalta ancora più vistosamente dal confronto con Ovidio. Orazio non fa parola di una connessione, già nota al rapsodo dell'inno omerico e denunciata vistosamente dal nome stesso di questa divinità nella religione romana e nelle pratiche del culto: la connessione tra Mercurius e la radice di mercari (il mondo delle merci, del mercimonium e dei mercatores) e l'idea del lucrum, del profitto economico, indissolubilmente legata a questa sfera dell'attività umana e della vita sociale.

Questa connessione era profondamente radicata nella concezione antica che squalificava il mercante come una figura sociale che ricava il proprio profitto senza aggiungere al valore-lavoro dell'oggetto nessun lavoro supplementare e quindi falsando inevitabilmente quello che sarebbe il giusto prezzo della merce. ${ }^{24}$ Il mercante costruisce il proprio profitto attraverso l'astuzia, l'inganno, la frode: quando non è propriamente un bugiardo e un simulatore è quanto meno un dissimulatore, uno che usa a suo vantaggio le informazioni di cui dispone in anticipo rispetto ai suoi interlocutori. Questa figura insidiosa tesse le trame del suo interesse contro gli interessi della comunità. Nella riflessione etico-politica di Cicerone, ${ }^{25}$ la mercatura (soprattutto il piccolo commercio al minuto) è una attività condannabile proprio in quanto dipende costituzionalmente dalla ragione astuta, dalla menzogna e dall'inganno. Solo in commercio su larga scala (magna mercatura) "è un'attività dai tempi lunghi, che giustificano il guadagno, e può pertanto essere praticata senza falsità (sine vanitate), il piccolo commmercio (tenuis mercatura) si fonda su un guadagno che è inevitabilmente frutto dell'inganno (de off. I,I5O-I5I)" ${ }^{26} \mathrm{Il}$ grande commerciante può aspirare a vedere riconosciuto il coraggio di chi affronta i pericoli del mare e una funzione civica di assicurare i rifornimenti alla città, ma un vero e proprio riconoscimento sociale avviene solo nel momento in cui egli è in grado di convertire le attività acquisitive nel possesso fondiario, l'unico che non lo porrà continuamente di fronte ai dilemmi del conflitto tra l'utile e l'honestum.

Quello che è davvero notevole nella rappresentazione ovidiana delle idi di maggio è che il calendario della città imperiale ospita la festa di un dio antichissimo e modernissimo capace di 'sdoganare' la figura del mercante senza nessun bisogno di ipocrisie e di sublimazioni. Il mercante che rivolge la sua preghiera a Mercurio non ha nessun imbarazzo a rivendicare il lucrum come una regola di vita e costruisce il proprio personaggio senza reticenza alcuna con gli stessi materiali che costituivano il cliché negativo del mercante ladro ingannatore e spergiuro. Soprattutto, non c'è alcun segnale di dissociazione moralistica da parte del poeta, nessuna presa di distanza:

24 Veyne (I976) II8ss.; Giardina (I986); Giardina (I989) 270ss.; Giardina (I994), che riprende vari interventi in materia dello stesso autore.

25 Narducci (1989) sopr. 234ss.

26 Giardina (I989) 28I. 


$$
\begin{aligned}
& \text { Ov. fast. 5,68I-690 } \\
& \text { "Ablue praeteriti periuria temporis", inquit } \\
& \text { "ablue praeteritae perfida verba die. } \\
& \text { sive ego te feci testem, falsove citavi } \\
& \text { non audituri numina vana Iovis, } \\
& \text { sive deum prudens alium divamve fefelli, } \\
& \text { abstulerint celeres improba dicta Noti: } \\
& \text { et pateant veniente die periuria nobis, } \\
& \text { nec curent superi siqua locutus ero. } \\
& \text { da modo lucra mihi, da facto gaudia lucro, } \\
& \text { et fac ut emptori verba dedisse iuvet." }
\end{aligned}
$$

La sfrontata preghiera che egli rivolge al suo dio richiama evidentemente la preghiera silenziosa e inconfessabile a Laverna che Orazio metteva sulle labbra del falso vir bonus:

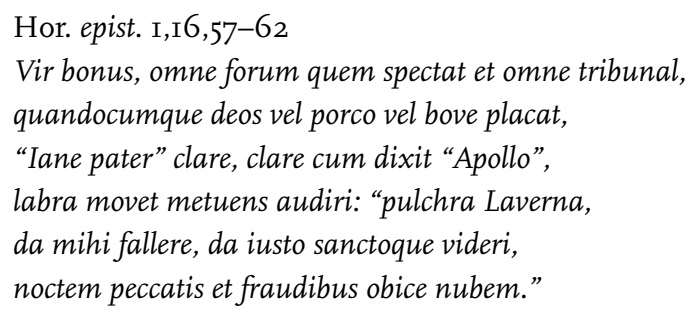

La complicità con cui il dio sorride dall'alto, riconoscendo nel mercante la realizzazione di se stesso come ladro, spergiuro e imbroglione, garantisce che la città imperiale può offrire un modello inclusivo che non lascia fuori nessuno, nemmeno le figure sociali e i comportamenti che nell'ottica del mos maiorum e della morale tradizionale non potevano trovare cittadinanza o per lo meno avevano bisogno di sublimazioni e di interpretazioni allegoriche.

La città imperiale è del resto un luogo che, se può essere attraversato da ladri, truffatori e spergiuri, non è tuttavia tale che i cives siano condannati a vivere nell'insicurezza e nelle turbolenze del passato. Lo spazio della città è ormai protetto stabilmente da una presenza insonne e capillare che assicura ordine e vigilanza. Nella riorganizzazione augustea dello spazio urbano che si celebra alle Calende di maggio con la festa dei Lares praestites, sono proprio i figli gemelli di Mercurio re dei ladri, quei Lares compitales le cui immagini sono associate ormai stabilmente nel culto al Genius principis, con il cane da guardia accovacciato ai loro piedi, ad assicurare ai cittadini tutta la protezione e le garanzie di cui essi hanno evidentemente bisogno:

Ov. fast. 5, 129-148

Praestitibus Maiae Laribus videre Kalendae aram constitui parvaque signa deum: voverat illa quidem Curius, sed multa vetustas destruit; et saxo longa senecta nocet. 
causa tamen positi fuerat cognominis illis

quod praestant oculis omnia tuta suis:

stant quoque pro nobis et praesunt moenibus Urbis,

et sunt praesentes auxiliumque ferunt.

at canis ante pedes saxo fabricatus eodem

stabat: quae standi cum Lare causa fuit?

servat uterque domum, domino quoque fidus uterque:

compita grata deo, compita grata cani.

exagitant et Lar et turba Diania fures:

pervigilantque Lares, pervigilantque canes.

bina gemellorum quaerebam signa deorum

viribus annosae facta caduca morae:

mille Lares Geniumque ducis, qui tradidit illos,

Urbs habet, et vici numina terna colunt.

La capitale dell'impero è anche il teatro di una continua, affascinante contesa tra guardie e ladri.

\section{Riferimenti bibliografici}

Barchiesi (I99I)

A. Barchiesi, "Discordant Muses", PCPhS 37, I-2I

Cassola (1975)

F. Cassola (a cura di), Inni omerici, Milano.

Combet Farnouz (1980)

B. Combet Farnouz, Mercure romain. Le culte public de Mercure et la fonction mercantile à Rome de la République archaiqque à l'époque augustéenne, Roma.

Combet Farnouz (198I)

B. Combet Farnouz, "Mercure romain, les 'Mercuriales' et l'institution du culte imperial sous le Principat augustéen”, ANRW II.I7.I, 457-50I.

Cristofoli (2008)

R. Cristofoli, Antonio e Cesare: anni 54-44, Roma.

Detienne e Vernant (1974)

M. Detienne e J.-P. Vernant, Les ruses de l'intelligence. La métis des Grecs, Paris (trad. it. Roma-Bari 1977).

Fantham (1983)

E. Fantham, "Sexual comedy in Ovid's Fasti: sources and motivations", HSCPh 87, I85-2I6. 


\section{Fraschetti (1990)}

A. Fraschetti, Roma e il principe, Roma-Bari.

\section{Giardina (1986)}

A. Giardina, "Le merci, il tempo, il silenzio. Ricerche su miti e valori sociali nel mondo grecoromano", Studi storici 27, 277-302.

\section{Giardina (1989)}

A. Giardina, "Il mercante”, in: Id. (a cura di), L'uomo romano, Roma-Bari, 270-298.

\section{Giardina (1994)}

A. Giardina, "Il mercante nel mondo romano", in: A. Giardina e A. J. Gurevič (a cura di), Il mercante dall'antichità al Medioevo, Roma-Bari, 4-59.

\section{Giardina (1997)}

A. Giardina, L'Italia Romana. Storie di un'identità incompiuta, Roma-Bari.

\section{Holleman (I973)}

A. W. J. Holleman, “Ovid and the Lupercalia”, Historia 22, 260-268.

\section{Labate (2010)}

M. Labate, Passato remoto. Età mitiche e identità augustea in Ovidio, Pisa-Roma.

\section{Littlewood (I975)}

R. J. Littlewood, "Ovid's Lupercalia (Fasti 2, 267-452). A study in the artistry of the Fasti", Latomus 34, 1060-1072.

\section{Miller (1993)}

J. F. Miller, "Ovidian Allusion and the Vocabulary of Memory", MD 30, I53-164, rist. in:

P. E. Knox (a cura di), Oxford Readings in Classical Studies: Ovid, Oxford 2006, 86-99.

\section{Narducci (1989)}

E. Narducci, Modelli etici e società. Un'idea di Cicerone, Pisa.

\section{Newlands (1995)}

C. Newlands, Playing with Time. Ovid and the Fasti, Ithaca and London.

\section{Nisbet-Hubbard (1970)}

R. G. Nisbet e M. Hubbard, A Commentary on Horace Odes, Book I, Oxford.

Perutelli (2006)

A. Perutelli, Ulisse nella cultura romana, Firenze.

\section{Scheid (1992)}

J. Scheid, "Myth, Cult and Reality in Ovid's Fasti", $P C P h S$ 38, II8--13I

\section{Stanford (1954)}

W. B. Stanford, The Ulysses Theme. A Study in the Adaptability of a Traditional Hero, Oxford.

Zecchini (200I)

G. Zecchini, Cesare e il mos maiorum, Stuttgart. 